\title{
THREE DIMENSIONAL INTERACTIVE GRAPHIC DISPLAY PROGRAM FOR MICHIGAN SAP (MSAP) $\dagger$
}

\author{
MOVSES J. KALDJIAN \\ Departments of Civil Engineering and Naval Architecture and Marine Engineering, College of Engineering, \\ The University of Michigan, Ann Arbor, MI 48109, U.S.A.
}

(Received 30 November 1975)

\begin{abstract}
To extend the usefulness of the Michigan Structural Analysis Program (MSAP), a computer graphics program called MSAPLOT has been developed. Using MSAP input data it plots line, plane and 3-D objects in isometric form as well as their orthotropic projection. MSAPLOT allows the user of MSAP to accomplish the following: (1) Check visually on the screen the correctness of the input data of the model being studied. (2) View the deformed structure. (3) Produce an ink drawing through the CalComp plotter. (4) Draw pictures of any object following input format of MSAP. Note that MSAPLOT is equally applicable to all input data of SOLID SAP and to most of SAP IV. Static analysis mode and cartesian coordinates are needed for the latter.
\end{abstract}

\section{INTRODUCTION}

The purpose of this paper is to describe the graphic program MSAPLOT to the user of MSAP which is a finite element program to perform structural analysis at the University of Michigan.

MSAPLOT allows a visual check of the discretized structural model using the input data of MSAP. This is important when large structural problems are being solved, or when the automatic element generation options of MSAP are used. A mistake in element definition could result in an unnecessary expenditure to the user.

MSAPLOT will also plot, if desired, the deformed structure after MSAP has been run.

An added feature of MSAPLOT is its ability to plot line, plane and 3-D objects in isometric form in addition to their orthotropic projections by simply following the input data format of MSAP.

MSAPLOT is a completely independent unit. The only thing it has in common with MSAP is its input data. Thus it can be used for graphics purposes by itself without any reference to MSAP.

Once the input data for MSAP is prepared for a given structure, the user calls for MSAPLOT from a graphic display terminal (Computek, Tektronix 4010, 4012, etc.) and sees whether his structure is modeled correctly. If not, he adjusts the input data to elininate the errors.

After the latter is accomplished, he is then ready to call on MSAP and obtain the displacements and the stresses throughout the structure.

Calling MSAPLOT after MSAP has been run, allows the user to see the deformed structure graphically. A hard copy of the initial or deformed structure in any orientation can be obtained, if desired, by calling on the CalComp plot option of the program's menu.

MSAPLOT is operational on an IBM/370-168 Computer at the University of Michigan. It utilizes two graphics libraries to manipulate and display various basic finite element patterns.

The first is the Michigan Graphics Interpreter [2] library (MGLIB). It is used to rotate the structure and to transform the 3-D graphical display.

†Presented at the Second National Symposium on Computerized Structural Analysis and Design at the School of Engineering and Applied Science, George Washington University, Washington D.C., 29-31 March 1976.
The second is the Michigan Basic Software library (CKLIB) [3] package for the Computek and Tektronix terminals. It is used to scale and to display the structure. CKLIB is also used by the command section of the program to interact with the user and to restore the menu and base view of the structure on the screen and help produce the CalComp plot.

MSAPLOT is written in standard FORTRAN IV, and is transferable to other computers.

No attempt will be made here to describe the MSAP program's element components nor its input data statements. For this the reader is referred to the SOLID SAP [1] manual published by the University of California, Berkeley. The latter is possible since MSAP and SOLID SAP have similar element libraries and more important, identical input data formats as far as MSAPLOT is concerned.

\section{DATA INPUT}

After the input data of the problem has been prepared according to MSAP (or SOLID SAP or SAP IV [4] in static mode) format, and is stored in a file, MSAPLOT may be run using the following Michigan Terminal System (MTS) commands:

\section{\$RUN CENA:MSAPLOT}

When execution begins the program will prompt the user for his data file name by printing on the screen,

\section{ENTER INPUT DATA FILE NAME.}

At this stage the user enters the name of the file in which the input data of his problem is stored. If the program is unable to obtain this file, it prints out the message,

\section{NAME.}

FILE NOT AVAILABLE, ENTER NEW FILE

On the other hand if the ENDFILE statement is encountered before all the input data is read, the program will print out the message,

ENDFILE ON INPUT, TRY " $N$ " FOR NEW FILE OR " $M$ " FOR MTS. 
For either of these messages the user should follow the instructions of the message which follows the comma. The first part of these messages describes the type of error encountered, and the remainder is a prompt for the user's action.

After the correct input data has been entered, the program prints out the following on the screen,

\section{IF DEFLECTED STRUCTURE TO BE DISPLAYED ENTER $Y$ OTHERWISE ENTER $N$.}

The user must enter the letter $Y$ or $N$ at this stage. If $Y$ is entered, the program prints out the message

\section{ENTER DEFLECTION DATA FILE NAME.}

If MSAPLOT is run immediately after running MSAP, the user must enter the temporary file name "-DEF" which contains all his deflection information. If "-DEF" has been copied into a permanent file, say "DEFLEC", then the latter may be entered at this time instead. "DEF" is a temporary file which was created automatically by MSAP to store the graphic display of the deflected structure. Unless it is copied into a permanent file it is destroyed after signing off.

After the deflection data file name is entered, the program will print out the message,

\section{ENTER MAGNIFICATION FACTOR}

The magnification factor is used to enlarge the value of deflections (displacements and/or rotations) and to make them visible.

If more than one load case, let us say $n$, had been considered at the same time the following message is printed out

\section{TOTAL NUMBER OF LOAD CASES $=n$. ENTER LOAD CASE.}

The user decides which load case he wants to see displayed and enters its number at this time.

If there are no major errors in the input and/or deflection data files, the program will display at this time the command section menu along with the $x-y$ plot of the structure under study.

MSAPLOT divides the screen into two display regions. Region 1 is used as a reference picture region where the first display is drawn. Region 2 is the main display area where the user can blow-up part of the structure or rotate the structure to obtain a different view with Region 1 coordinate axes as reference. The reference picture may also be rotated if desired. Unless the reference picture in Region 1 is altered the first view of the structure shown there, i.e. the $x-y$ plot, will be seen displayed throughout.

If there is any gross error in the data file the program will either not display at all or display something quite different. For trouble shooting purposes the user may list "-GDAT" after the program has turned control over to MTS. File "-GDAT" is a temporary file created automatically by MSAPLOT. It contains the general nodal point and element information from the input data of the structure. The user may check the list of "-GDAT" to find where he had gone wrong, correct his input file and try again.

\section{COMMAND MODE}

When the program has completed processing the input data, the command section menu along with the $x-y$ plot of the structure in Region 1 is displayed on the screen. It is now ready to receive orders. In this mode, the presence of the graphic cursor (or cross hairs) on the screen indicates that the program is waiting for the user to enter a command.

The commands are divided into three groups. The first group is the display manipulation commands. This is followed by subcommands to indicate to the program the parameters of the specified operation. The second group is the program control commands, and is followed by the third group, the auxiliary commands.

A detailed description of these commands is presented next.

\section{$3.1 \mathrm{~S}$-Section}

The display manipulation command $\mathbf{S}$ enables the user to select a part of the structure enclosed by a rectangle from Region 1 or Region 2, and cnlarge it in Region 2. The parameters of a section to be blown-up are the lower-left-hand corner and the upper-right-hand corner respectively of the rectangle. This is done by moving the cursor (or cross hair) first to the lower corner and entering the region number from which the section is being taken ( 1 for Region 1 and 2 for Region 2), then moving the cursor to the upper corner and entering the region number as before. If the second region number is not the same as the first region number, the program will request the user to respecify both corners again.

The program ignores all characters except 1 and 2 , when specifying the first corner. After both corners have been specified with the same region number, the program outlines the rectangle to be enlarged and awaits for the user's command to display it in Region 2.

If the user is not satisfied with the choice of his rectangle he may enter 0 (zero) at this time to cancel the enlarging operation and may start all over again, otherwise he enters again the region number from which the rectangle is being taken. Current displays only may be shown when attempting to enlarge a section from Region 1.

\section{$3.2 Q$ or $\boldsymbol{R}-$ Rotate}

The display manipulation commands $\mathbf{Q}$ or $\mathbf{R}$ are used to perform rotations on the structure to obtain a different viewing angle. All rotations are relative to the base picture shown in Region 1. Entering $\mathbf{Q}$ displays the rotated picture in Region 1, and entering $R$ displays it in Region 2. After $\mathbf{Q}$ or $\mathbf{R}$ has been entered, the program awaits for the command parameter $W$ to be entered. The Command 0 (zero) may be entered to cancel the commands $\mathbf{Q}$ or $\mathbf{R}$ at this time if desired. All characters except $W$ and 0 (zero) are ignored by the program at this stage.

After $\mathbf{W}$ has been entered, the program will prompt the user for the value of the angles in degrees to be rotated about the $x, y$ and $z$ axes respectively. Rotations are always specified with respect to the current Region 1 display axes.

\section{$3.3 \mathrm{M}$-Call MTS}

$\mathbf{M}$ is a program control command, with it the user can stop the execution of the program and return control to MTS. The program may be restarted with the MTS command \$RESTART. 


\section{$3.4 \mathrm{~N}-\mathrm{New}$ file}

The control command $\mathbf{N}$ causes a return to the input section of the main program and prompting begins again. The user can enter this command and display more than one structure without having to call MSAPLOT again.

\section{$3.50-$ Cancel}

The auxiliary command $\mathbf{0}$ (zero) is used to return to command mode from one of display manipulation commands without changing the current display.

\subsection{P-Save Region 1}

The first display shown of a structure is automatically made to be the base picture after a new file name is entered. The auxiliary command $P$ is used to keep the base picture in Region 1, unchanged. It is used only if Region 1 has been rotated from its initial display by using the $\mathbf{Q}$ command.

\subsection{T-New magnif}

The program control command $\mathbf{T}$ is used to allow a change in the magnification factor of the displacements, i.e. when the user is not satisfied with his current magnification factor effects on the deformed structure.

\section{$3.8 U-N e w$ load}

The program control command $U$ is used to display various load cases when more than one loading is considered in the structural analysis.

\subsection{C-CalComp}

The program control command $\mathbf{C}$ is used to produce a CalComp plot. When the user is satisfied with the display on the screen and would like to have a hard copy made through the University of Michigan CalComp plotter he enters the command $\mathbf{C}$.

The program then will prompt him for the scale factor of his CalComp plot. This scale can be any number greater than 0.0 and less than or equal to 3.5. The display seen on the screen has a scale factor of 1.0 .

After entering the scale factor, the user, to obtain a CalComp plot, returns control to MTS by entering the command $\mathbf{M}$ and proceeds as described below.

3.9.1. When the user enters the $\mathbf{C}$ command, the data for CalComp is automatically generated by MSAPLOT and stored in a temporary file called "-OBUF". Note that this file is destroyed at signoff.

3.9.2. If the user would like to view what he has placed in the file "-OBUF" he may enter the following command:

\section{\$RUN *PLOTSEE EXECUTION BEGINS ENTER FILE NAME}

$$
\begin{aligned}
& \text { (MTS reply) } \\
& \text { (MTS question) }
\end{aligned}
$$

The user should enter at this time the file name "-OBUF".

The program then displays the user's CalComp plot for his inspection. When user is ready to inspect his next plot, if such is the case, he should enter at this time the $\mathbf{N}$ command. MTS will respond by printing again,

\section{ENTER FILE NAME}

(MTS question)

User enters his next file name. To terminate this sequence, he should enter

\footnotetext{
†This must be a permanent file name.
}

\section{SENDFILE}

(or CONTROL-C)

3.9.3. If user decides to obtain a CalComp plot, he should enter the following statements,

\section{SCREATE FILE1 $\dagger$ \\ \$COPY -OBUF FILE1 \\ \$PERMIT FILE1 RO \\ \$RUN *CCQUEUE \\ EXECUTION BEGINS \\ ENTER FILE NAME}

(MTS reply)

(MTS question)

The user then enters his file name

\section{FILE1}

MTS will now give the user the approximate plotting time for the CalComp plot. If user wants the plot(s) he should reply with a $\mathbf{Y}$, otherwise reply with a $\mathbf{N}$. If user confirms the plot, MTS will give him the receipt number for his plot ...... Write this number down and don't lose it!

MTS will then come back with

\section{ENTER FILE NAME}

(MTS question)

If user has another file to be plotted, he may enter it at this time, otherwise he should reply with

\section{\$ENDFILE}

(or CONTROL-C)

which will cause the execution to be terminated.

\section{SUMMARY}

The importance of MSAPLOT is determined by the size and type of the structure which MSAP is being called upon to solve. For small simple plane problems, unless the user wants a hard copy picture of the discretized model or the deformed structure, it is probably not very exciting. For three dimensional or large plane problems, however, it is a very convenient means of checking the input data before running the more costly MSAP program. This is especially important when the automatic element generator option of MSAP is used.

MSAPLOT is a graphics tool for the user of MSAP. It enables him to do the following.

(1) To check visually and very economically the input data of the model being studied on the screen. In this way, errors can be detected and corrected before the expensive operation of running MSAP.

(2) To see what the deformed structure looks like after running MSAP.

(3) To produce an ink drawing through the CalComp plotter in any desired deformation size and orientation.

As an added feature, MSAPLOT may be used also to draw pictures of any line, plane and 3-D object by simply following the input data format of MSAP.

Acknowledgements-The author wishes to acknowledge the assistance of Kiang Ning Huang in preparing this program and the financial support in part of the National Science Foundation, Grant NSF-67 2898. The Master's degree project on graphics of V. Anthony Currie and some work done by J. R. Obermark were utilized as background material in developing the present graphics program package. 


\section{REFERENCES}

1. E. L. Wilson, SOLID SAP - a static analysis program for three dimensional solid structures. UC-SESM 71-19. Dept. of Civil Engng, Univ. of Calif., Berkeley (Dec. 1972).

2. J. Van Roekel, MGI user's guide-an introduction to the Michigan graphics interpreter. Dept. of Aerospace Engineering, Univ. of Michigan (Aug. 1973).

3. J.W. Conklin and M. Barnett, A basic software package for the computek terminal on MTS.

4. K. J. Bathe, E. L. Wilson and F. E. Peterson, SAP IV-a structural analysis program for static and dynamic response of linear systems. EERC 73-11. Earthquake Engng Research Center, College of Engng, Univ. of Calif., Berkeley (June 1973).

\section{APPENDLX}

Examples

The illustrations that follow demonstrate in some detail how the MSAPLOT program is run. It is assumed that all necessary input and deflection data have been prepared and obtained, and are stored in permanent files.

Step 1

$$
\text { SIGNON ID PW }=\ldots \quad \text { (CR) } \dagger \quad \text { USER }
$$$$
\text { MTS's response }
$$

Step 2

RUN CENA:MSAPLOT

EXECUTION BEGINS

ENTER DATA FILE NAME

Step 3

PIPE (3-D Clutch Input Data File)

\section{IF DEFLECTED STRUCTURE TO} ENTER $Y$ OTHERWISE ENTER $N$

Step 4

USER

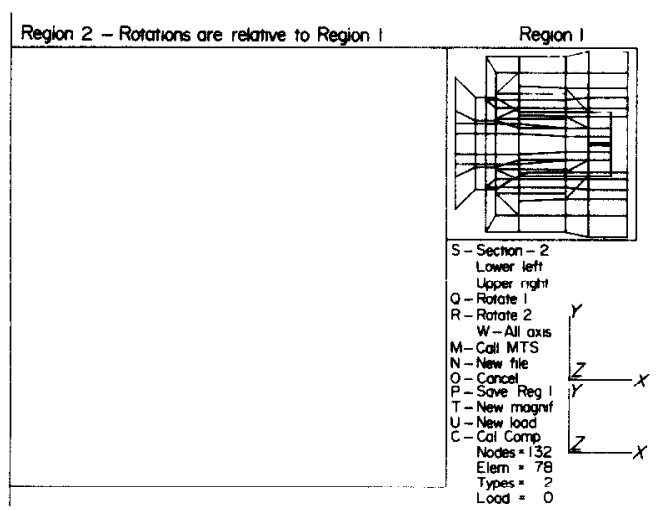

Fig. 1. Cantilever clutch coupling CL-41900. 8. Nodal point 3-D element.

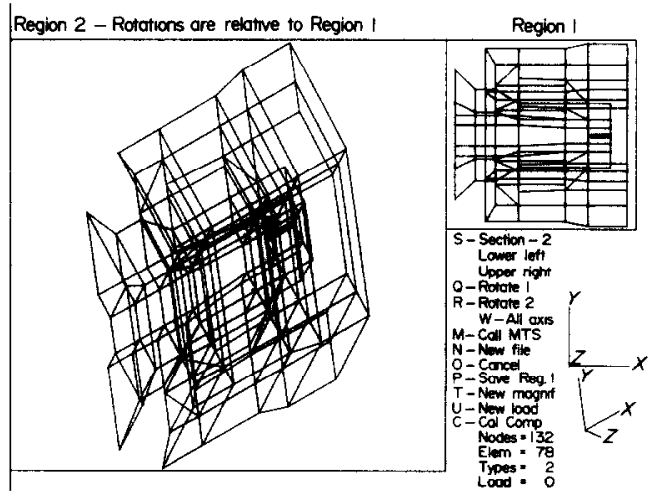

Fig. 2. Cantilever clutch coupling CL-41900. 8. Nodal point 3-D element.
MSAPLOT will now display Fig. 1 on the screen. Note that the reference plot on the upper right hand corner REG. 1 , is the $x-y$ projection of the 3-D structure.

From the menu shown in Fig. 1 enter

$\mathbf{R}$ (Wait for crosshair to appear)

USER

$\mathbf{W}$

$$
\text { ANGLE? }
$$

USER

$30,45,30$

MSAPLOT

USER

MSAPLOT now displays Fig. 2 on the screen. The main display area (Region 2), shows an isometric plot of the structure according to the angles specified above. Note that the reference picture in REG. 1 remained unchanged.

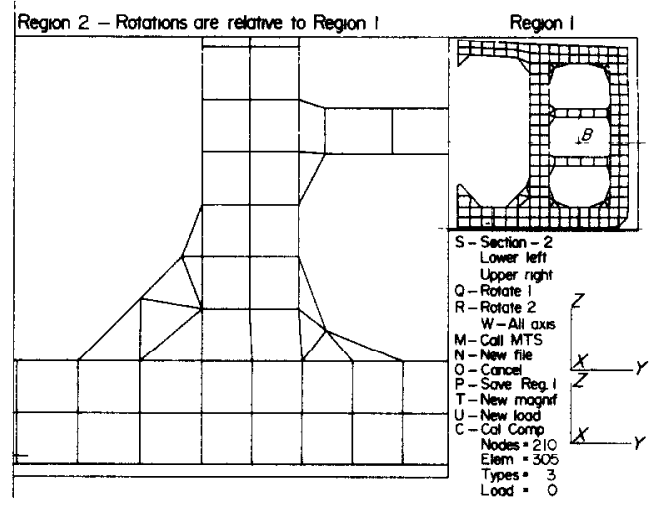

Fig. 3. Analysis of tanker transverse web frame gross mesh.

A midship section is defined in the $y-z$ plane, using a 2-D finite element model. In the initial display a vertical line alone described this structure in REG. 1. Figure 3 displays the midship section after the following commands are performed.
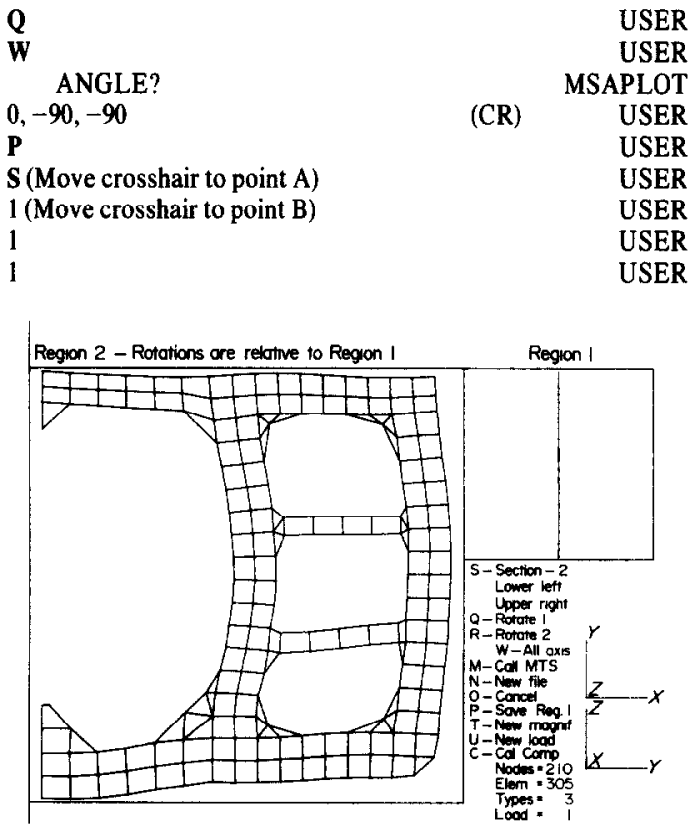

Fig. 4. Analysis of tanker transverse web frame gross mesh.

The deflected midship section for a magnification factor of 100 is shown in Fig. 4. The following commands were performed for this display. 


\section{ANGLE?}

$0,90,90$

\section{MSAPLOT} USER

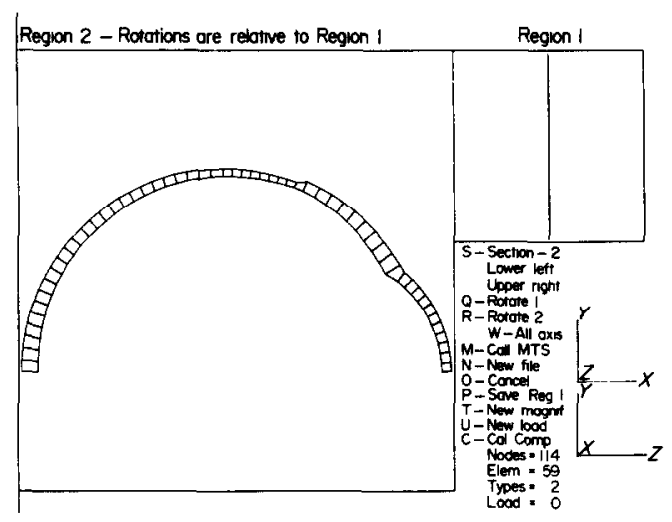

Fig. 5. Finite element model of the eyeball.

A finite element model of the eyeball using axisymmetry and defined in the $y-z$ plane is shown in Fig. 5. The following commands were performed to obtain the display.
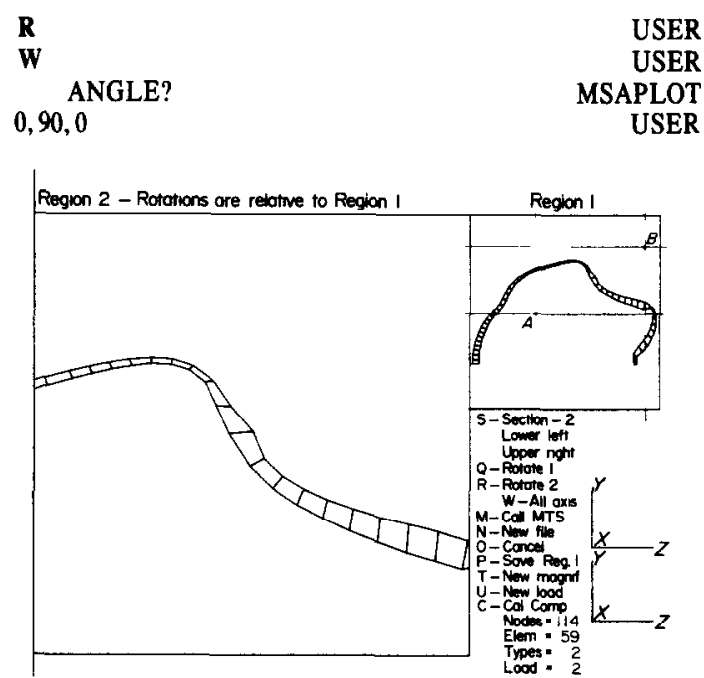

Fig. 6. Finite element model of the eyeball.

The deflected eyeball model is shown in Fig. 6 for load case 2 and a magnification factor of 1 . The following commands were performed.

$\mathbf{Q}$

\section{ANGLE?}

$0,90,0$

P

S (Move crosshair to point A)

1 (Move crosshair to point $B$ ) 1
USER

USER

MSAPLOT

USER

USER

USER

USER

USER

USER
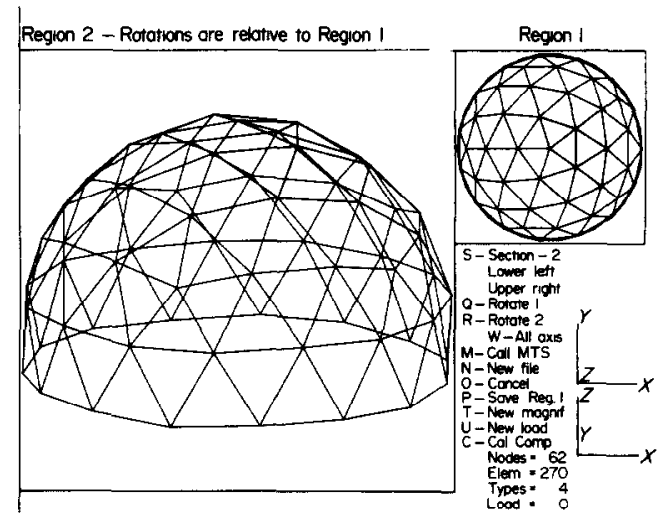

Fig. 7. Geodesic dome analysis.

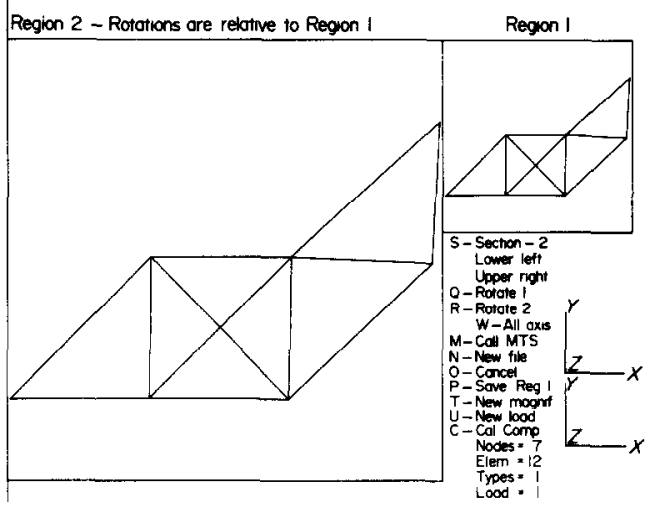

Fig. 8. Elevated truss.

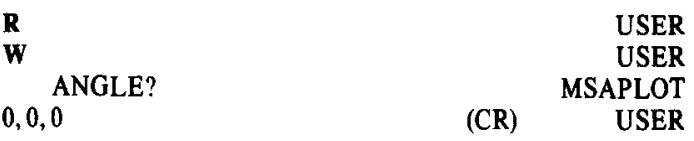

Figure 7 shows a finite element model of a geodesic dome using 3-D frame and membrane elements. The commands

\section{$\mathbf{R}$}

$\mathbf{W}$ ANGLE?
USER

USER MSAPLOT

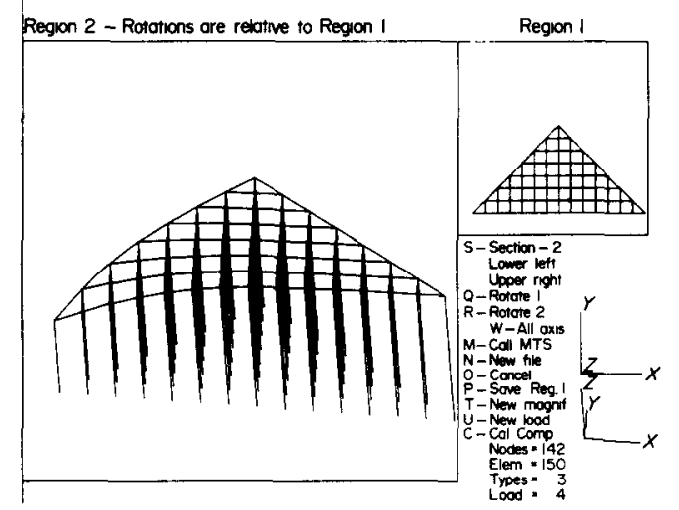

Fig. 9. Shell elem. wedge on elas $\mathrm{fdn}$ (truss $\mathrm{fdn}$ ), load conc/press at/ near apex.

In Fig. 9 a finite element model of a shell (plate) wedge on elastic foundation is shown. Load case 4 was used along with a magnification factor of 50. The following commands were preformed to obtain this display.

$\mathbf{W}$

USER

USER

A deflected 2-D simple truss model is shown in Fig. 8. The magnification factor used was 10 . The commands performed were,

ANGLE?

$-60,0,4$ 